\title{
(t)
}

\section{LA NUEVA REVOLUCIÓN TECNOLÓGICA Y SUS IMPACTOS A LA SALUD DE LOS TRABAJADORES}

\author{
The new technological revolution and its impacts \\ on Worker's health
}

\section{Guillermo Foladori ${ }^{1}$}

\section{RESUMEN}

Las nanotecnologías constituyen una nueva revolución tecnológica que se impone rapidamente a nivel mundial. Se trata de una revolución en la forma de manipular la materia. Su presencia está en prácticamente todos los sectores económicos y muchos productos ya están en el mercado. Varias nanopartículas y nanoestructuras, que constituyen la base de esta revolución tecnológica, han demostrado ser tóxicas en estudios in vitro o en animales de laboratorio. Los productos de la nanotecnología que se lanzan al mercado no son sometidos a estudios de toxicidad. Como resultado, trabajadores y consumidores están expuestos y son sujeto de riesgo, al igual que los ecosistemas que sean alcanzados. Este artículo explica en qué consisten las nanotecnologías y sus implicaciones para los trabajadores.

\section{PALABRAS CLAVE}

Nanotecnología. Trabajo. Revolución tecnológica. Riesgos.

\section{ABSTRACT}

Nanotechnology constitutes a new technological revolution that rapidly stands out at a global level. It is a revolution in the

\footnotetext{
${ }^{1}$ Economista. Professor do Doutorado em Estudos do Desenvolvimento, Universidade Autonóma de Zacatecas, México. E-mail: <gfoladori@gmail.com>.
} 
way to manipulate matter. Its presence is basically in every economic sector, and many of its products are already in the market. Several nanoparticles and nanostructures, which are the basis of this technological revolution, have shown to be toxic in studies in vitro and tests on laboratory animals. Nanotechnology products released in the market do not undergo toxicity testing. As a result, workers and consumers are exposed to these toxicity risks, as well as the ecosystems the products reach. This article aims at explaining what nanotechnology consists of and its consequences to workers.

\section{KEYWORDS}

Nanotechnology. Labor. Technological revolution. Risks.

\section{LAS NANOTECNOLOGÍAS: UNA NUEVA REVOLUCIÓN TECNOLÓGICA}

Los sindicatos y organizaciones sociales no terminan de entender las implicaciones de una tecnología cuando el capital está introduciendo una nueva en los procesos productivos. Este es el caso con las nanotecnologías. Se desconocen las implicaciones de los organismos genéticamente modificados a la salud humana y el medio ambiente, y falta por investigar los efectos a la salud de cientos de químicos que ya están en el mercado cuando surgen las nanotecnologías con sus nuevas implicaciones.

La nanotecnología es la manipulación de la materia a escala atómica y molecular. Esto ya se logra con la precisión suficiente como para elaborar nanopartículas de diferentes materiales que son incorporadas a procesos productivos. Muchos productos que ya están en el mercado contienen nanopartículas. Hay alimentos, cosméticos, medicinas, vestimenta, maquinaria, cemento, artículos deportivos, armas, etc. Las nanotecnologías representan una nueva revolución industrial por varias razones; entre ellas:

1) Porque el material en nanoescala manifiesta propiedades diferentes a las que ofrece la misma materia en tamaño mayor. Así por ejemplo, el oro, que no es reactivo en los tamaños en que lo conocemos, en nanoescala se convierte en reactivo y puede ser utilizado como sensor. El grafito, como lo conocemos en los lápices, es un material blando; pero el mismo trabajado en nanoescala como tubo de carbono es 100 veces más duro que el 
acero. Los elementos químicos cambian su resistencia, flexibilidad, conductividad eléctrica, magnetismo, etc., cuando están en tamaños menores a los 100 nanómetros. ${ }^{2}$ Un nanómetro es la mil millonésima parte de un metro (10-9), y 60 nanómetros pueden ser el diámetro de un virus. Una célula humana puede ser 20 mil veces mayor a una nanopartícula, por lo cual estas últimas pueden introducirse fácilmente a través de la piel e inclusive ser "tragadas" por las células. En muchas ocasiones primero se conocen las propiedades "nuevas" de un material, para después buscar en qué aplicarlas. Para la ciencia es como descubrir un mundo nuevo de materiales. Para el capital es una nueva frontera de valorización.

2) Porque a medida que disminuye el tamaño de la materia la superficie externa en relación a su masa es mayor, lo cual hace que la reactividad de una nanopartícula sea mayor que la reactividad del mismo material en mayores tamaños. Esto permite que menos materia cumpla, en algunos casos, la misma función para la cual antes se requería de más materia. Así, por ejemplo, un fármaco nanoencapsulado requieren de mucho menos producto químico que el mismo fármaco en las tradicionales píldoras. Lo mismo sucede con los pesticidas en la agricultura, que nanoencapsulados ahorran materia prima. Otra manera de economizar en materia es el caso de los productos que son insolubles, y que al disminuirlos a nanopartículas su distribución en los productos que los contienen es más homogénea. Esto es muy utilizado en alimentos a los cuales se agregan saborizantes, homogeneizadores, anticoagulantes, fortificantes o colorantes, así como en pesticidas. Aún otra ventaja del tamaño nano es que el producto se vuelve más bioasimilable. Por todas estas razones las nanotecnologías significan una economía de materiales para el capital y, en muchos casos, una ventaja técnica.

3) Porque al tratarse de una revolución en la materia, a diferencia de otras revoluciones tecnológicas donde el cambio se da en la fuente de energía o en un proceso mecánico o electrónico, afecta todas las ramas de la producción y sectores económicos (¡no hay ninguna o sector

\footnotetext{
${ }^{2}$ Se trata de una cifra convencional, ya que algunos materiales manifiestan propiedades "nuevas" con 300 y aún con 1000 nanómetros.
} 
que no utilice materia!) y, por tanto, tiene un horizonte de mercado mucho más amplio, en lugar de introducirse de un sector hacia otro como ha sucedido con las revoluciones tecnológicas anteriores.

4) Por último, porque por desarrollarse en un momento histórico en que la economía está totalmente globalizada, la velocidad con que se introduce en los diferentes países y regiones del mundo es más rápida que lo que sucedió con las tecnologías anteriores.

En la alimentación, la nanotecnología se aplica en los productos, en los envases, en suplementos alimenticios y en la producción agrícola. Hay más de 200 compañías que investigan y/o producen en la rama. Se utiliza la nanotecnología en el propio producto, para, por ejemplo, homogeneizar la textura y enfatizar el sabor en cremas y helados, o para reducir el contenido graso, como lo investigan Kraft, Unilever o Nestlé. O, también, para agregarle al producto suplementos alimenticios nanoencapsulados, como Omega3, fortificantes o adelgazantes. También se investiga en alimentos que llevan incorporados cosméticos, como lo hace L'Óreal en asociación con Nestlé, o BASF. Se utiliza la nanotecnología en los envases, para hacer más durable el producto en los anaqueles de los supermercados, como la cerveza en botella de nano-cerámica de la Miller Brewing; o para que la materia prima no se deteriore, como experimenta McDonalds o Mr. Kipling. Las grandes corporaciones químicas de semillas, como Syngenta, Monsanto, Bayer y Dow Chemicals investigan y producen agroquímicos y semillas nanoencapsuladas (FOLADORI, 2012).

La rama de cosméticos es posiblemente donde hay más productos de la nanotecnología en el mercado. La gran mayoría de las corporaciones transnacionales tienen cremas antiarrugas, filtros solares y shampoo, como Chanel, Clinique, L'Oreal, Revlon, Johnson \& Johnson, Proctor \& Gamble, o Lancome. Aplicada a los filtros solares, la nanoescala hace a la crema transparente, evitando el tradicional color blanco. También se usa la nanotecnología para difundir la luz y ocultar arrugas, y muchas otras funciones (FOLADORI; INVERNIZZI, 2012). Hay cepillos de diente y pasta dentífrica con nanopartículas de plata como bactericida. La Unión Europea está legislando sobre el uso de nanotecnología en cosméticos, dada la gran cantidad de evidencias de los efectos perjudiciales sobre la salud. 
Varios electrodomésticos incluyen nanopartículas de plata como bactericida, como en el caso de aires acondicionados, refrigeradores, lavarropas y lavavajillas de Samsung o LG. Filmes de nanotecnología son usados para cubrir pisos, o nanopartículas son incorporadas a pinturas y también en aerosoles para aplicar en muebles y pisos. Vidrios son procesados con nanotecnología para evitar que se adhiera polvo y suciedad y para facilitar el escurrimiento del agua. En textiles, la aplicación de técnicas nanotecnológicas evita que la ropa se manche y se arrugue. En algunos casos incorporan nanopartículas de plata con efecto bactericida en uniformes médicos o en vestimenta especial para enfermos, pero también en sábanas, toallas y medias. Medicinas procesadas con nanotecnología prometen ser más eficientes y tener menos efectos colaterales. La nanotecnología está presente en artículos deportivos, en raquetas de tenis, palos de golf, zapatos deportivos, ropa climatizada, etc. Las principales marcas de computadores, celulares, y juegos electrónicos almacenan su información en baterías de litio con ánodos recubiertos con nanotecnología, y utilizan nano dispositivos electromecánicos. Automóviles de lujo ya llevan incorporadas más de 30 partes que contienen nanodispositivos o combinan nanopartículas.

La industria de armamento es una de las que más se ha beneficiado y más impulsa las nanotecnologías. De misiles de precisión a superexplosivos, y de sensores a chalecos antibala, el interés militar está ligado al avance de las nanotecnologías.

Es claro que las nanotecnologías se introducen en el mercado de la mano de la propaganda optimista que las presenta como la solución a la mayoría de los problemas del mundo. Nos dicen que la nanotecnología facilitará el uso de energía solar y alternativas no contaminantes, permitirá producir más eficientemente en la agricultura aumentando la oferta regular de alimentos, medicinas manipuladas con nanotecnología evitarán epidemias y enfermedades antes no tratadas, y así por delante. Pero esto no es de sorprender porque ha sido el discurso tradicional que acompaña cada revolución tecnológica. Los trabajadores y consumidores, sin embargo, están preocupados con los efectos perjudiciales y resultados imprevistos. 


\section{IMPLICACIONES DE LAS NANOTECNOLOGÍAS PARA LA SALUD HUMANA Y EL MEDIO AMBIENTE}

Uno de los aspectos que el capital no ha asumido a la velocidad con que pone los productos de las nanotecnologías en el mercado, son los efectos de estos procesos productivos y sus productos en la salud humana y de los ecosistemas. Esto es grave, porque se ha demostrado que muchas nanopartículas son tóxicas en animales de laboratorio y en análisis in vitro, con lo cual es muy probable que también lo sean para los trabajadores que entran en contacto con ellas, para otros organismos vivos y, también, para los consumidores. Además, se sabe que los trabajadores están expuestos al contacto con nanopartículas, debido al hecho de que los controles y medidas de precaución que se utilizan en los laboratorios e industrias no son eficientes contra las nanopartículas. Así, por ejemplo, una máscara de protección contra el polvo no evita la penetración de nanopartículas. Nanopartículas suspendidas en líquido pueden atravesar sin dificultad los guantes de latex que comúnmente se emplean en laboratorios. Veamos estos dos aspectos: efectos tóxicos y exposición.

\subsection{Las nanopartículas pueden tener efectos tóxicos aún cuando el mismo elemento en tamaño mayor no sea tóxico}

Los productos con nanotecnología entran al mercado sin ningún estudio de toxicidad. El argumento de científicos, empresarios y gobierno para continuar incentivando el desarrollo de las nanotecnologías y su presencia en el mercado sin estudios de toxicidad previos, es que la información existente sobre toxicidad de las nanoparticulas a la salud humana no es aún concluyente. Se trata de un argumento parcial e interesado, que explota la novedad del tema sin dar importancia a la salud de trabajadores y consumidores. Si se sitúa la afirmación en el contexto de lo que ya se sabe sobre toxicidad de las nanopartículas la afirmación es encubridora de potenciales riesgos.

Existe suficiente información como para tener una actitud precautoria; la que no se aplica al poner por delante los intereses del mercado, que es lo que actualmente sucede. Esta información puede resumirse en: 
- Las nanopartículas manufacturadas tienen tamaño, forma, y propiedades físico-químicas y biológicas (toxicológicas) novedosas. Son estas propiedades novedosas las que dan a las nanotecnologías su gran potencial, ya que la materia desarrolla manifestaciones que no son conocidas en tamaño mayor. Si son tratados como materiales nuevos para aplicaciones prácticas, debieran ser tratados con el mismo respeto en términos toxicológicos, realizando estudios sobre los efectos de las nanopartículas en la salud humana, elaborando guías de manejo obligatorias basadas en el grado de exposición y riesgo, estableciendo canales de información a los trabajadores, y, evitando lanzar al mercado productos sin previo análisis toxicológico de su ciclo de vida. Como ejemplo de la desconsideración de los impactos sobre la salud, baste decir que para fines del 2010 los expertos del National Institute for Occupational Safety and Health (NIOSH) de los Estados Unidos señalaban que no se habían realizado estudios sobre potenciales efectos adversos a la salud en trabajadores que producen nanotubos de carbono o nanofibras, una materia prima que crece exponencialmente en el mercado de nano-materias primas y que se aplica a muy diversos sectores económicos (NATIONAL INSTITUTE FOR OCCUPATIONAL SAFETY AND HEALTH, 2010).

- Varias nanopartículas han resultado tóxicas en análisis in vitro, y en organismos vivos. El NIOSH viene insistiendo, desde el año 2005, sobre la necesidad que los trabajadores de empresas que manipulan dióxido de titanio en polvo ( $\mathrm{TiO}_{2}$ ) tengan condiciones de seguridad diferentes según se trate de partículas de más de 100 nanómetros o de menos. Es decir, recomiendan tratar al $\mathrm{TiO}_{2}$ nanoparticulado (menos de $100 \mathrm{~nm}$ ) como si fuese un producto diferente del no nanoparticulado. Este producto se utiliza en fábricas de pintura, cosméticos, plásticos, papel, alimento. Desde 1988 NIOSH lo clasificó como cancerígeno, y la inhalación como principal vía de penetración al organismo. Estudios en animales han mostrado inflamación pulmonar y tumores, tanto para los polvos nanoparticulados como con partículas de mayores tamaños (MURASHOV, 2011).

Los nanotubos de carbono, cuya producción ha crecido exponencialmente en la última década, y donde su costo por gramo ha pasado de cien mil dólares a centavos, y se aplica en decenas de ramas económicas (e.g. electrónica, baterías, células solares, plás- 
ticos, polímeros, sensores, dispositivos médicos) es otro ejemplo de la desconsideración por los riesgos a la salud y el medio ambiente de la política de nanotecnología. La NIOSH sugiere un enfoque precautorio, ya que constata que diversos estudios en animales muestran fibrosis pulmonar, inflamación por granulomas y facilidad de migrar hacia la pleura (NATIONAL INSTITUTE FOR OCCUPATIONAL SAFETY AND HEALTH, 2010). Los nanotubos de carbono se comportan de manera muy similar al amianto que, según la Organización Mundial de la Salud, provoca, aún hoy en día, 90 mil muertos por año; y se sigue produciendo en varios países, entre ellos Brasil. ${ }^{3}$ Se sabe que los trabajadores de fábricas que producen y/o emplean nanotubos de carbono o nanofibras de carbono están expuestos, pero no hay estudios de los efectos en la salud (NATIONAL INSTITUTE FOR OCCUPATIONAL SAFETY AND HEALTH, 2010). Si esto sucede en los Estados Unidos, ¿cuáles serán las condiciones de trabajo en los países menos desarrollados?, donde también se producen nanotubos de carbono, muchas veces en condiciones de seguridad inferiores.

Una compilación de 1991 a noviembre de 2010 de NanoCeo, un banco de artículos científicos no exhaustivo sobre riesgos relacionados a las nanotecnologías, da el siguiente resultado: 176 artículos científicos relacionados con riesgos de los nanotubos de carbono, 216 de riesgos de las nanopartículas de plata, 81 de las nanopartículas de titanio, 72 de fullerenos y buckyballs, y 49 de puntos cuánticos, todos ellos nanopartículas o nanoestructuras de entre las más comunes en los productos del mercado. Otra institución que reúne información sobre los riesgos asociados con nanopartículas es el Consejo Internacional de Nanotecnología (ICON), que forma parte de la Universidad de Rice en Estados Unidos. De 2000 a 2010 su base de datos registró un aumento en los trabajos científicos sobre los riesgos para la salud humana y el medio ambiente de las nanotecnologías que pasó de alrededor de un centenar en 2005 a más de 550 en 2010. ${ }^{4}$ Estas compilaciones de artículos científicos

\footnotetext{
${ }^{3}$ En febrero 2012 dos empresarios de fábricas de asbestos en Italia (Eternit) fueron sentenciados a 16 años de cárcel y más de 100 millones de euros en multa por la muerte de más de 2000 obreros en sus fabricas (CARLILE, 2012).

${ }^{4}$ Base de datos ICON propia fusión de los siguientes nanomateriales: Carbono o Metal u Orgánicos / Polímeros o Semiconductores o de Óxido o Múltiple u Otras / No Especificadas] + Peligro para los siguientes grupos de los trabajadores [In-
} 
dan una clara idea de que hay suficiente información científica para tomar una actitud precautoria.

\subsection{Trabajadores y consumidores están expuestos a potenciales riesgos de las nanopartículas}

El NIOSH reconoce que los mayores riesgos de las nanopartículas están en polvos en estado sólido, dispersas o aglomeradas en los polvos, por ejemplo en cosméticos. En un segundo nivel de riesgo están las suspendidas en líquidos, como los nanotubos en agua. En un tercer nivel están las fijas en matrices, como los filmes delgados. Las que, por último, ofrecerían menor riesgo son las incorporadas en nanoestructuras, como aleaciones en metales.

Esta escala de riesgo se asocia, también, a las diferentes maneras en que las nanopartículas pueden ingresar al organismo e interactuar con éste. En términos generales las principales vías de ingreso potencial de nanopartículas al organismo son la inhalación, la ingestión y la penetración a través de la piel. Cuando se trata de productos médicos con nanopartículas también pueden ingresar al organismo vía inyección o por el desprendimiento de nanopartículas utilizadas en implantes. Ciertamente, también es necesario llevar en cuenta el caso de accidentes, como incendios o explosiones, que pueden extender los riesgos de las nanopartículas a personas sin protección (MAYNARD et al., 2006).

La exposición a las nanopartículas es diferente según su ciclo de vida y la manera cómo los trabajadores y consumidores se relacionan con él. En la etapa de investigación, por ejemplo, los propios investigadores son sujeto de riesgo; pero también los trabajadores de servicio de mantenimiento de los laboratorios, y quienes se encargan de manipular los desechos. Cuando se producen nanomateriales como materia prima en masa, los sujetos directamente expuestos son investigadores, técnicos y operarios de las empresas, y también los encargados de almacenar el producto y los transportistas que llevan la nano-materia prima a las industrias que las requieren. En las industrias que incorporan nano-materia prima a sus procesos los obreros están expuestos, y, tal vez, también el

dustriales / Trabajadores de Investigación o Los consumidores o la Población en General o los Ecosistemas u Otro / No Especificado] + Artículos de Revistas por Revisar + Ingeniería. Disponible en: <http://icon.rice.edu/>. 
personal administrativo y de servicio (SCHULTE et al., 2008). Según el tipo de producto y la forma de uso, el consumidor final está expuesto a las nanoparticulas. Al final del ciclo de vida y en los procesos de desecho, limpieza y transporte, el medio ambiente puede verse afectado.

Las tres afirmaciones: las nanopartículas tienen efectos toxicológicos nuevos, las nanopartíuclas son tóxicas in vitro y para diversos organismos vivos en laboratorio, $y$, trabajadores y consumidores están expuestos, son suficiente parar tener un enfoque precautorio. La respuesta de empresas y muchos científicos es que no se ha comprobado toxicidad en humanos; además de que las nanopartículas no se desprenden de la matriz a la cual están incorporadas, por lo que los estudios in vitro y con otros seres vivos no son representativos del riesgo. Pero, de lo que se trata es de prevenir en lugar de esperar que ocurran implicaciones a la salud humana, y, el argumento de que no se desprenden de la matriz es relativo. En el caso de los cosméticos, las nanopartículas penetran directamente por la piel, en textiles el uso, lavado y manipuleo desprende nanopartículas, en alimento e utensilios de cocina pueden ser absorbidas directamente o por desprendimiento, en dispositivos médicos, interactúan con el organismo; y, en todos los casos los artículos que contienen nanopartículas tienen un ciclo de vida útil, y cuando éste finaliza pasan a tener efectos inciertos sobre los ecosistemas y las cadenas tróficas.

\section{CONCLUSIONES}

Las nanotecnologías representan una nueva revolución industrial que se está imponiendo. No sólo los países desarrollados investigan y producen con nanotecnología, también muchos en desarroIlo, como es el caso, en América Latina, de Brasil, México, Argentina, Colombia, Venezuela, Cuba, etc.

Las nanopartículas y nanoestructuras tienen la peculiaridad de que manifiestan propiedades físico-químicas diferentes a las que los mismos elementos químicos muestran en escala mayor. Pero, al hacerlo, también manifiestan propiedades biológicas y, consecuentemente, toxicológicas diferentes. Esto debiera obligar a un cuidadoso estudio de toxicidad antes de enviar un producto de la nanotecnología al mercado. Este no es, infelizmente, el caso. Por el contrario las empresas vienen introduciendo productos en el mer- 
cado desde principios de este siglo de forma sistemática y creciente, sin ningún tipo de control de toxicidad ni de regulación. Esto significa un riesgo para los trabajadores que producen nanomaterias prima o las manipulan, pero también para los consumidores, muchos de los cuales entran en contacto directo a través del producto en el mercado, y para los ecosistemas que reciben los residuos o pueden ser espacios de accidentes.

\section{REFERENCIAS}

CARLYLE, Erin. Swiss billionaire sentenced to 16 years In Asbestos Case. Forbes, 15 feb. 2012. Disponible: <http://www.forbes.com/ sites/erincarlyle/2012/02/15/swiss-billionaire-sentenced-to-16-yearsin-asbestos-case/>.

FOLADORI, G. Trabajadores y nanotecnología. Observatorio del desarrollo: Investigación, reflexión y análisis, v. 1, n. 3, 2012. Disponible: <http://estudiosdeldesarrollo.net/observatorio/ob3/6.pdf>.

FOLADORI; INVERNIZZI, N. Implicaciones sociales y ambientales del desarrollo de las nanotecnologías en América Latina y el Caribe. Zacatecas (México): ReLANS; Curitiba (Brasil): IPEN, 2012. Disponible: <http://www.ipen.org/pdfs/Nanotecnologia_es.pdf>.

MAYNARD, Andrew D. et al. Safe handling of nanotechnology. Nature, v. 444, p. 267-269, 16 nov. 2006.

MURASHOV, Vladimir. Titanium Dioxide: a Changing Paradigm in Occupational Risk Management. Centers for Diseade Control and Prevention, The National Institute for Occupational Safety and Health (NIOSH) Science Blog, 9 may 2011. Available from: <http:// blogs.cdc.gov/niosh-science-blog/2011/05/tio2/>. Accessed on: 16 abr. 2012.

NATIONAL INSTITUTE FOR OCCUPATIONAL SAFETY AND HEALTH (USA). Occupational Exposure to Carbon Nanotubes and Nanofibers. NIOSH Current Intelligence Bulletin, nov. 2010. Available from: <http://www.cdc.gov/niosh/docket/archive/pdfs/NIOSH-161-A/0161A-110110-DraftDocument.pdf>. Accessed on: 15 mar. 2012.

SCHULTE, Paul et al. Occupational Risk Management of Engineered Nanoparticles. Journal of Occupational and Environmental Hygiene, London, v. 5, n. 4, p. 239-249, apr. 2008. 